Reprod. Nutr. Dévelop., 1985, 25 (1 A), 75-81.

\title{
Action de la méthionine alimentaire sur la morphogenèse appendiculaire d'Artemia. Hypothèse sur l'évolution du phénotype de ce crustacé
}

\author{
Arantxa HERNANDORENA
}

Laboratoire du Muséum National d'Histoire Naturelle, C.E.R.S., Plateau de l'Atalaye, 64200 Biarritz, France.

Summary. Action of dietary methionine on the appendicular morphogenesis in Artemia. Hypothesis on the phenotypical evolution of this crustacean.

Artemia larvae reared in a folic acid-free, thymidine-free, methionine-rich, nutritive medium develop three instead of eleven pairs of thoracic appendages. Present and previous results show that morphogenetic potentialities can be reduced or increased by the nutritional conditions prevailing during larval life. A model has been proposed which would explain the evolution of the Artemia phenotype.

\section{Introduction.}

Au cours de la vie larvaire d'Artemia, on assiste à la morphogenèse des deux yeux composés et des onze paires d'appendices thoraciques. Grâce à l'utilisation d'un milieu nutritif synthétique défini pour l'élevage axénique de ce crustacé, la nature de certains facteurs nutritifs capables de modifier la morphogenèse oculaire et appendiculaire a pu être définie. II s'agit du tryptophane, de l'acide folique et des dérivés puriques et pyrimidiques. L'excès alimentaire de tryptophane induit la phénocopie de la mutation garnet qui affecte la structure et la couleur des yeux d'Artemia (Hernandorena, 1984). La double carence en acide folique et en thymidine induit une métamorphose précoce empêchant la morphogenèse des appendices postérieurs qui demeurent à l'état d'ébauches rudimentaires (Hernandorena, 1970a, 1979a). L'excès alimentaire d'acide cytidylique par rapport à la concentration du milieu en acide adénylique induit la morphogenèse de gonopodes surnuméraires sur les segments abdominaux normalement apodes (Hernandorena, 1970a, 1977, 1979b).

Comme le montrent les résultats reportés ici, la méthionine alimentaire vient s'ajouter à la liste des facteurs nutritifs capables de modifier la morphogenèse appendiculaire d'Artemia. 


\section{Matériel et méthodes.}

La race Utah et la méthode mise au point par Provasoli et d'Agostino (1969) pour l'élevage axénique de cette race sont utilisées.

Les concentrations des différents facteurs nutritifs sont exprimées en $\mathrm{mg}$ pour $100 \mathrm{ml}$ de milieu nutritif. Le beoin alimentaire des larves en dérivés puriques est satisfait par l'acide adénylique $(60 \mathrm{mg}$ d'AMP pour $100 \mathrm{ml}$ de milieu) et le besoin en dérivés pyrimidiques par l'acide cytidylique $(20 \mathrm{mg}$ de CMP pour $100 \mathrm{ml}$ de milieu). Le besoin alimentaire des larves en thymidine est facultatif chez les larves élevées dans un milieu nutritif contenant de l'acide folique (Hernandorena, 1970b).

L'action de la L-méthionine à des concentrations comprises entre 0 et $50 \mathrm{mg}$ pour $100 \mathrm{ml}$ de milieu est étudiée chez les larves élevées dans un milieu nutritif contenant soit de l'acide folique $(1,4 \mathrm{mg}$ pour $100 \mathrm{ml}$ de milieu) soit de la thymidine $(10 \mathrm{mg}$ pour $100 \mathrm{ml}$ de milieu), soit carencé en acide folique et en thymidine. Dans les conditions d'élevage standard, le besoin alimentaire des larves en méthionine est satisfait par des particules d'albumine d'œuf. Les concentrations de méthionine libre viennent s'ajouter à celle contenue dans l'albumine $(20 \mathrm{mg}$ pour $100 \mathrm{ml}$ de milieu).

Un index numérique basé sur les changements morphologiques qui accompagnent les mues a été défini par Provasoli et d'Agostino (1969) pour évaluer la vitesse de croissance. La correspondance entre les stades larvaires et la morphogenèse appendiculaire est reportée dans le tableau 1. Les stades 5-6 et 7-8 sont regroupés car leur détermination nécessite un examen microscopique, examen difficile dans les conditions d'élevage axéniques si les animaux ne sont pas sacrifiés pour cet examen. A partir de la $8^{e}$ mue, la détermination des stades peut se faire à l'aide d'une loupe à main. L'index 7 a été attribué à la 9 e mue (Hernandorena, 1970a).

La métamorphose dont le premier signe visible à l'œil nu est la rotation des antennes survient après la morphogenèse de onze paires d'appendices thoraciques $\left(11^{e}\right.$ mue : index 10 , fin de la vie larvaire). La vie juvénile se caractérise par I'allomorphose antennaire, la formation des gonopodes sur les segments génitaux et l'allongement de l'abdomen $\left(12^{\circ}\right.$ mue : index 11). L'index de croissance 12 est attribué aux jeunes adultes chez lesquels le dimorphisme antennaire permet de caractériser le sexe à l'œil nu.

TABLEAU 1

Correspondance entre mues larvaires et morphogenèse appendiculaire.

\begin{tabular}{|c|c|c|c|c|c|}
\hline Stades .. & $5-6$ & $7-8$ & 9 & 10 & 11 \\
\hline Index $\ldots \ldots \ldots \ldots \ldots$ & 4 & $5-6$ & 7 & 8 & 10 \\
\hline Nombre d'appendices formés $\ldots \ldots \ldots \ldots \ldots \ldots$ & 0 & 3 & 6 & 8 & 11 \\
\hline
\end{tabular}




\section{Résultats.}

On sait que la morphogenèse appendiculaire des larves élevées dans un milieu nutritif contenant soit de l'acide folique soit de la thymidine est normale (Hernandorena, 1970b). Dans ces conditions l'addition de méthionine à des concentrations comprises entre 0 et $50 \mathrm{mg}$ pour $100 \mathrm{ml}$ de milieu ne modifie pas la morphogenèse.

Contrairement aux larves élevées dans un milieu contenant de l'acide folique et carencé en thymidine, les larves élevées dans un milieu carencé en acide folique et contenant de la thymidine additionné ou non de méthionine $n^{\prime}$ atteignent pas le stade de la maturité sexuelle. A partir de la $12^{\mathrm{e}}$ mue, ces animaux gisent au fond des tubes où ils peuvent survivre plusieurs jours.

Chez les larves élevées dans un milieu nutritif carencé en acide folique et en thymidine et contenant $60 \mathrm{mg}$ d'AMP et $20 \mathrm{mg}$ de CMP pour $100 \mathrm{ml}$ de milieu, la métamorphose survient après la morphogenèse de six paires d'appendices, c'està-dire à la 9 e mue. Dans ces conditions, l'addition de méthionine à des concentrations égales ou supérieures à $10 \mathrm{mg}$ pour $100 \mathrm{ml}$ de milieu induit la métamorphose dès la $8^{\ominus}$ mue alors que seules trois paires d'appendices sont formées. Cette morphogenèse est illustrée dans la figure 1. Les appendices 4-5-6 et les bourgeons 7-8 sont rudimentaires, les antennes ont subi l'allomorphose caractéristique de la vie juvénile et l'abdomen s'est allongé. Le caractère ơ des antennes est reconnaissable.

Dans les milieux nutritifs non additionnés de méthionine ou contenant 5 et $10 \mathrm{mg}$ de méthionine pour $100 \mathrm{ml}$ de milieu, $100 \%$ des larves atteignent le stade de I'allomorphose antennaire. Dans les milieux contenant 25 et $50 \mathrm{mg}$ de méthionine pour $100 \mathrm{ml}$ de milieu, ce pourcentage est réduit à $70 \%$.

L'examen microscopique des larves élevées dans les milieux carencés en acide folique et en thymidine additionnés de méthionine révèle les mêmes symptômes que ceux décrits antérieurement pour les larves élevées dans des milieux carencés en acide folique et en thymidine mais non additionnés de méthionine (Hernandorena, 1970a, b). Le cycle de mue est interrompu en $D_{2}$, les cellules phagocytaires de réserves sont surchargées de réserves lipidiques, le nombre de corpuscules sanguins en circulation est réduit et les mouvements des appendices manquent de coordination. La pigmentation des yeux semble anormale et les bourgeons appendiculaires sont mélanisés. Ces deux derniers symptômes ne se manifestent pas chez tous les individus.

\section{Discussion.}

Les résultats montrent que chez les larves élevées dans un milieu nutritif carencé en acide folique et en thymidine et additionné de méthionine, la morphogenèse appendiculaire réalisée avant la métamorphose correspond à celle de la $8^{e}$ mue larvaire. La réalisation des potentialités morphogénétiques des bourgeons appendiculaires qui précède la métamorphose est réduite à la morphogenèse de 


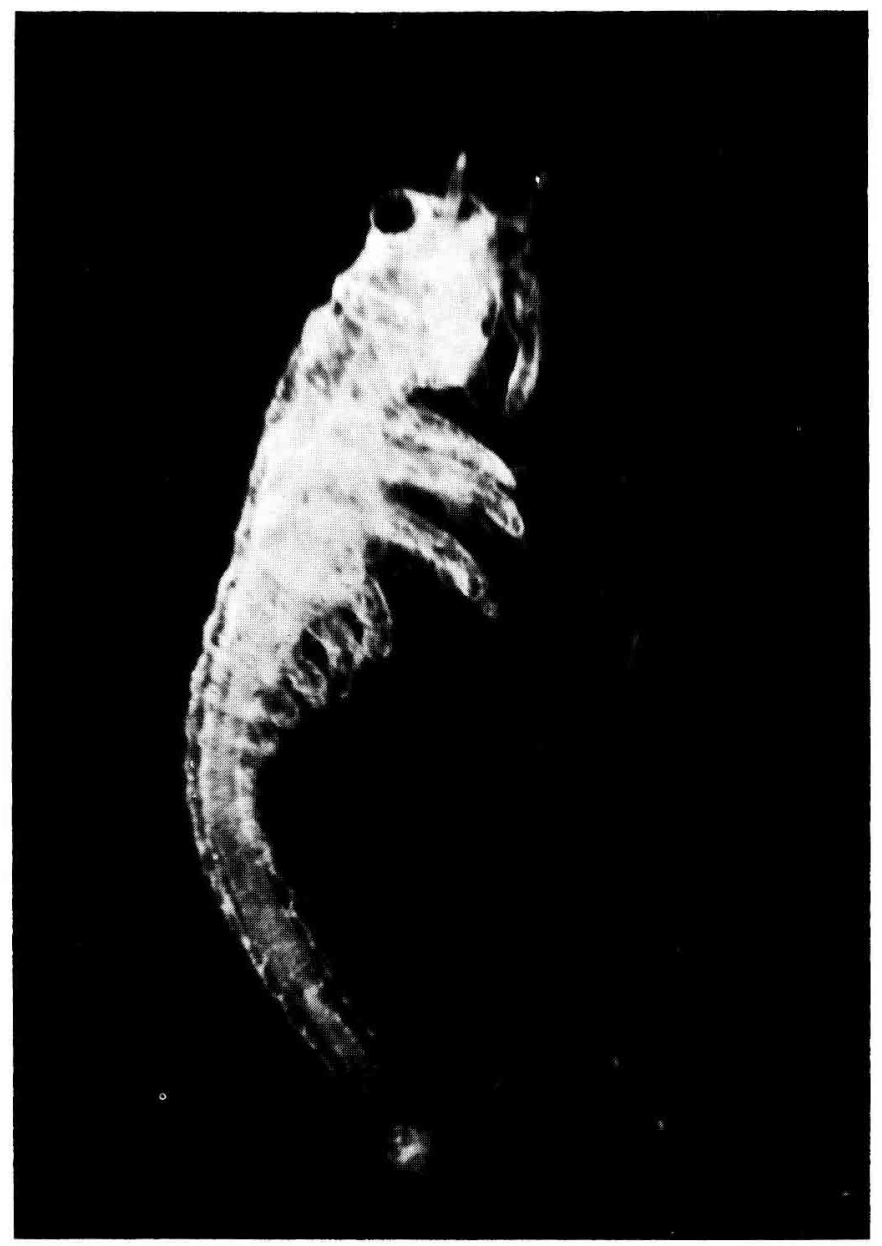

FIG. 1. - Morphogenèse appendiculaire correspondant à la $8^{e}$ mue chez un individu ayant subi l'allomorphose antennaire, obtenue dans un milieu nutritif carencé en acide folique et en thymidine et contenant $25 \mathrm{mg} \%$ de méthionine.

trois paires d'appendices thoraciques. La méthionine vient s'ajouter à la liste des facteurs nutritifs susceptibles de réduire les potentialités morphogénétiques.

Un schéma récapitulatif des différents effets morphogénétiques obtenus chez Artemia en modifiant la composition du milieu nutritif est proposé (fig. 2).

FIG. 2. - Représentation schématique des versions plus ou moins intégrales du programme de morphogenese appendicularre postembryonnaire d'Artemia. Les antennules, les antennes et les yeux composés sont schématiquement figurés.

$\mathrm{N}=$ Morphogenèse appendiculaire normale. $\mathrm{T}_{1-11}$ : segments thoraciques ; $\mathrm{G}_{1-2}$ : segments génitaux ; $A_{1-5}$ : segments abdominaux « normalement » apodes ; TS : telson.

$\mathrm{R}_{1-3}=$ Réduction des potentialités morphogénétiques.

$\mathrm{A}_{1-3}=$ Augmentation des potentialités morphogénétiques $\times$ appendices abdominaux lovisac médio-ventral chez la $Q$, gonopodes latéraux-ventraux chez le $\left.0^{*}\right\}$.

$\mathrm{PA}=$ phénotype archaıque. 

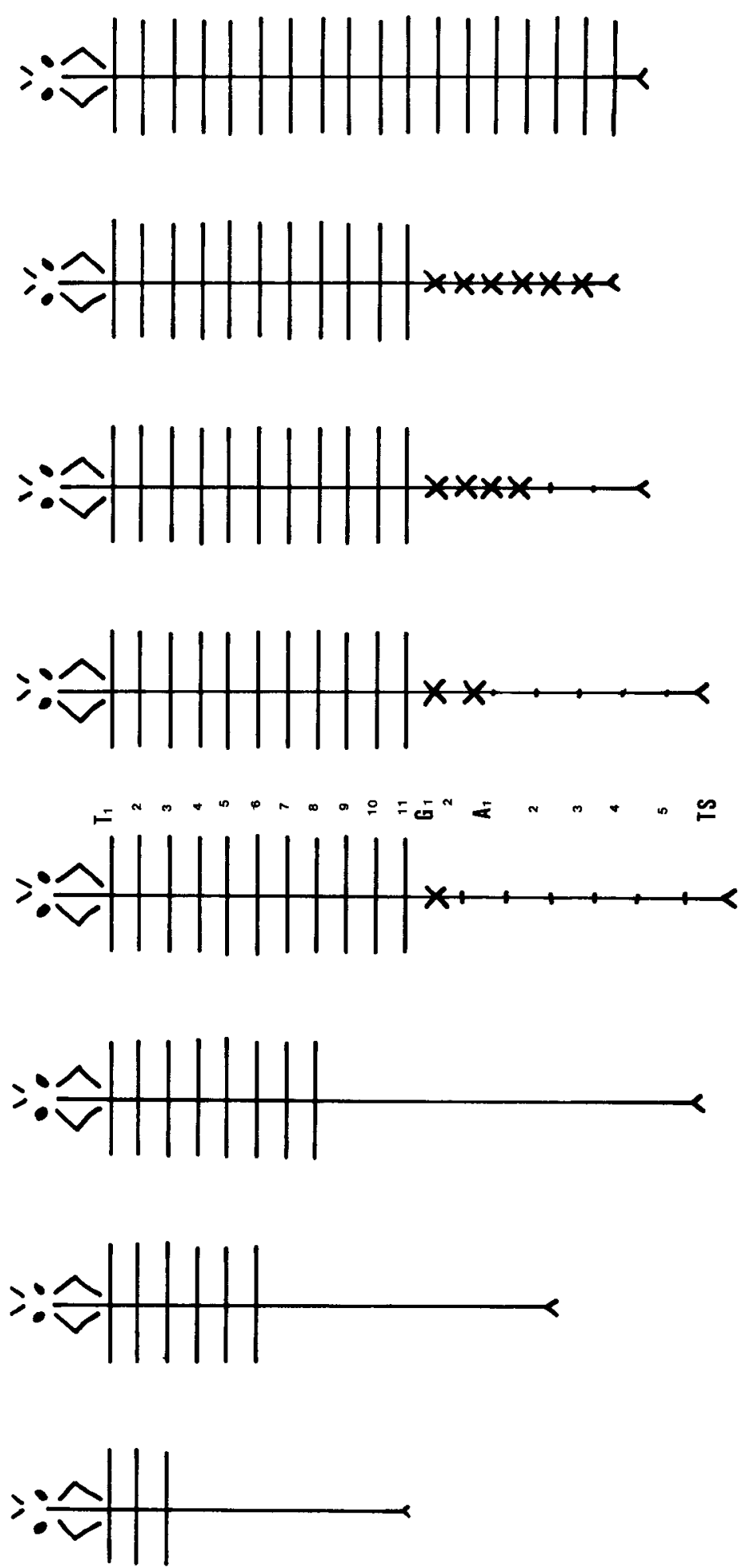

Reproduction, Nutrition, Développement $n^{\circ}$ 1A 1985. -6 $\varangle$

a

$\varepsilon^{2}$

๕

$\bar{a}$

$\mathbf{z}$

$\ddot{x}$

$\tilde{x}$

$\bar{x}$ 
L'effet morphogénétique de la carence alimentaire en acide folique dépend de la concentration du milieu en thymidine. La morphogenèse des larves élevées dans un milieu nutritif carencé en acide folique et non déficient en thymidine est normale : la métamorphose survient à la $11^{\mathrm{e}}$ mue après la morphogenèse de onze paires d'appendices thoraciques (fig. 2 : N). Chez les larves élevées dans un milieu carencé en acide folique et déficient en thymidine, la morphogenèse appendiculaire réalisée avant la métamorphose correspond à celle de la $10^{\mathrm{e}}$ mue $(\mathrm{Her}-$ nandorena, 1970a) (fig. $2: R_{3}$ ). Si le milieu est carencé en acide folique et en thymidine, elle correspond à celle de la $9^{e}$ mue (Hernandorena, 1970a) (fig. 2 : $R_{2}$ ).

L'effet morphogénétique de la double carence en acide folique et en thymidine dépend de la concentration du milieu en nucléotides purique et pyrimidique et en méthionine. Quand la concentration en nucléotides augmente ( $\mathrm{AMP}=100 \mathrm{mg} \% ; \mathrm{CMP}>20 \mathrm{mg} \%$ ), la morphogenèse appendiculaire réalisée avant la métamorphose correspond à celle de la $8^{\mathrm{e}}$ mue (Hernandorena, 1979a). Le même effet est obtenu chez les larves élevées dans un milieu non enrichi en nucléotides mais additionné de méthionine (fig. $2: R_{1}$ ).

$J$ 'ai défini les conditions nutritionnelles qui permettent d'induire des gonopodes surnuméraires sur les segments abdominaux normalement apodes, c'est-àdire les conditions qui permettent d'augmenter les potentialités morphogénétiques par rapport à celles exprimées chez les animaux élevés dans le milieu témoin (Hernandorena, 1970a, 1977, 1979b) (fig. 2 : $A_{1-3}$ ). La description de mosaïques par Bowen et al. (1966) permet d'établir I'homologie entre les appendices des segments thoraciques et ceux des segments génitaux.

L'examen du schéma récapitulatif montre que le programme de morphogenèse appendiculaire postembryonnaire d'Artemia peut être interprété dans des versions plus ou moins intégrales selon la composition du milieu nutritif dans lequel les larves sont élevées. La version " normale » ou contemporaine du programme de morphogenèse pourrait correspondre à une évolution régressive par rapport à la version " intégrale » qui reproduirait un phénotype archaïque caractérisé par la morphogenèse d'appendices sur tous les segments (fig. 2 : P.A.). Artemia aurait perdu au cours de son évolution les capacités de biosynthèse nécessaires à la réalisation du phénotype archaïque, capacités qui peuvent être partiellement restaurées dans les conditions du laboratoire par une alimentation riche en acide cytidylique. Artemia n'a aucune chance de rencontrer dans la nature les conditions, qui d'après l'hypothèse proposée, lui permettraient de ressembler à ses « ancêtres ». Par contre, Artemia trouve dans le milieu naturel, l'acide folique et la thymidine nécessaires à la réalisation du phénotype « sauvage », phénotype dont la normalité est une notion arbitraire.

\section{Conclusion.}

Les résultats obtenus chez Artemia montrent qu'à partir d'un même génotype, différents phénotypes sont obtenus en fonction de la composition du milieu nutritif. La version phénotypique du génotype d'Artemia serait la résultante 
des conditions métaboliques subies pendant la vie larvaire. Ces conditions dépendent des capacités de biosynthèse régies par le génotype et de la composition du milieu nutritif en acide folique, en dérivés puriques et pyrimidiques et en méthionine.

Reçu en mars 1984.

Accepté en septembre 1984.

\section{Références}

BOWEN S. T., HANSON J., DOWLING P., POON M., 1966. The genetics of Artemia salina VI. Summary of mutations. Biol. Bull., 131, 230-250.

HERNANDORENA A., 1970a. Obtention de morphogenèses appendiculaires abortives et surnuméraires chez Artemia salina (L.) (Crustacé Branchiopode) par carences alimentaires de base pyrimidique et de nucléotide purique. C. R. Acad. Sci. Paris, 271, 1406-1409.

HERNANDORENA A., 1970b. Action de deux inhibiteurs d'enzymes intervenant dans le métabolisme des ptérines chez Artemia salina (L.) (Phyllopoda) en milieu axénique. Crustaceana, 19 (1), 49-58.

HERNANDORENA A., 1977. Action antagoniste de nucléotides purique et pyrimidique alimentaires sur la morphogenèse appendiculaire d'Artemia salina (L.) (Crustacé, Branchiopode). C. R. Acad. Sci. Paris, 284, 1337-1339.

HERNANDORENA A., 1979a. Influence of dietary purines and pyrimidines on Artemia postembryonic development, 233-298. In Biochemistry of Artemia development. S. C. BAGSHAW et A. H. WARNER éd., Univ. Microfilms Int.

HERNANDORENA A., 1979b. Relationships between purine and pyrimidine dietary requirements and Artemia salina morphogenesis. Comp. Biochem. Physiol., 62 B, 7-12.

HERNANDORENA A., 1984. Induction by excess dietary tryptophan of a phenocopy in Artemia. Comp. Biochem. Physiol., 78 A, 327-331.

PROVASOLI L., d'AGOSTINO A., 1969. Development of artificial media for Artemia salina. Biol. Bull., 136, 434-453. 